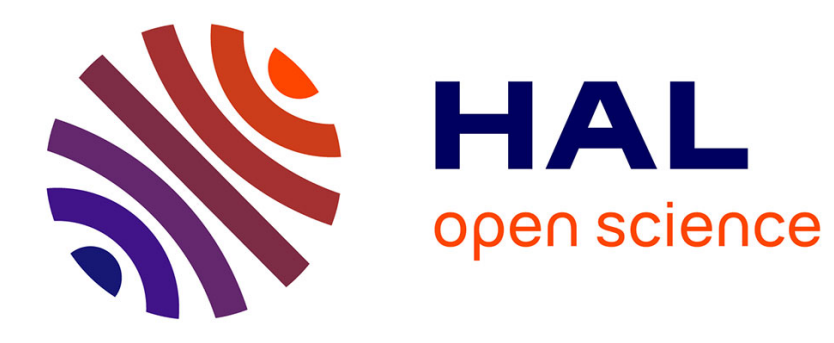

\title{
The Method of Tree-hypersequents for Modal Propositional Logic
}

Francesca Poggiolesi

\section{To cite this version:}

Francesca Poggiolesi. The Method of Tree-hypersequents for Modal Propositional Logic. David Makinson, Jacek Malinowski, Heinrich Wansing. Towards Mathematical Philosophy, 28, Springer, pp.31-51, 2009, Trends in logic, 978-1-4020-9083-7. 10.1007/978-1-4020-9084-4_3 . halshs-00775815

\section{HAL Id: halshs-00775815 https://shs.hal.science/halshs-00775815}

Submitted on 6 Sep 2018

HAL is a multi-disciplinary open access archive for the deposit and dissemination of scientific research documents, whether they are published or not. The documents may come from teaching and research institutions in France or abroad, or from public or private research centers.
L'archive ouverte pluridisciplinaire HAL, est destinée au dépôt et à la diffusion de documents scientifiques de niveau recherche, publiés ou non, émanant des établissements d'enseignement et de recherche français ou étrangers, des laboratoires publics ou privés. 


\title{
F. Poggiolesi The Method of Tree-hypersequents for Modal Propositional Logic
}

\begin{abstract}
In this paper we present a method, that we call the tree-hypersequent method, for generating contraction-free and cut-free sequent calculi for modal propositional logics. We show how this method works for the systems $\mathrm{K}, \mathrm{KD}, \mathrm{K} 4$ and $\mathrm{KD}$, by giving a sequent calculus for these systems which are normally presented in the Hilbert style, and by proving all the main results in a purely syntactical way.

Keywords: Contraction-free, Cut-free, Hypersequents, Modal logic, Sequent Calculus, Tree-hypersequents.
\end{abstract}

\section{Introduction}

One of the open problems of modal propositional logic consists in the lack of a good sequent calculus for (at least) its main systems, where we understand a good sequent calculus to be one that satisfies certain requirements, mainly listed by [1], [4] and [12], the principal ones being ${ }^{1}$ :

Subformula Property: we should be able to associate to every proof $d$ of the sequent calculus, a proof $d^{*}$ of the same final sequent, in which each formula is a subformula of the formulas occurring in the final sequent.

Semantic Purity: the sequent calculus should not make any use of explicit semantic elements, such as possible words or truth values.

Explicitness: logical rules should exhibit the constant they introduce only in the conclusion.

Separation: logical rules should not exhibit any constant other than the one they introduce.

Symmetry: each constant of the language of the sequent calculus should have at least two logical rules: one which introduces it on the left side of the sequent, one which introduces it on the right.

\footnotetext{
${ }^{1}$ The list is by no means exhaustive but our aim here is not to discuss the properties which define a good sequent calculus.

David Makinson, Jacek Malinowski and Heinrich Wansing (eds.),

Trends in Logic: Towards Mathematical Philosophy 
Invertibility: for each of the rules of the calculus it should hold that not only the conclusion is derivable from the premise(s), but also the premise(s) from the conclusion.

The first attempts made at solving such a problem use the standard sequent calculus (see, for example, [3], [6] and [11]), but they are generally not good sequent calculi, as they do not satisfy the subformula property and their rules are not explicit, nor invertible, nor separate nor symmetric.

More recently research has been oriented towards finding methods which can generate extensions of the standard sequent calculus. These methods can be divided in two groups: in one group there are methods which generate purely syntactic sequent calculi, the most important of which are the method of hypersequents [1] and the method of display logic [12]. In the other group there are methods which extend the standard sequent calculus by adding explicit semantic parameters. Some examples of this group are the calculus given by G. Mints [7] and the calculus given by S. Negri [8]. None of the methods proposed so far can generate a calculus which satisfies all the desired properties: the calculi of the second group for the very reason that they include explicit semantic parameters, and the calculi of the first group because they lack other properties normally required.

When this work was essentially completed, we were informed of the existence of a further method, created by R. Kashima [5], and then developed by K. Brünnler [2], which is called nested-sequents method (by Kashima), or deep-sequents method (by Brünnler), and which makes use of the same notion of sequent that we will present in this article, though using a different notation. The main difference between the nested (or deep) - sequents method and the one that we will introduce below, consists in the fact that here the proof of cut-elimination is developed in a purely syntactic way (see section 5), as well as the proof of admissibility of the several structural rules (see section 3), while Kashima and Brünnler use semantics instead (Brünnler sketches a syntactic cut-elimination procedure for the system $K$ in his paper). Moreover we apply the method for capturing the $D$ - axiom, while Kashima and Brünnler do not. Finally, although Kashima's calculi are not contraction-free and invertible, ours (and those of Brünnler) are.

We want also to point out that although the nested (deep, tree-hyper) - sequents method has doubtless several common points with the methods of Negri and Mints, it also has one important difference: it does not use any labels. A clarification about the property of "not using labels" and the advantages of having such property can be found in [2]. 
Having clarified the relationships with the recent related works, we can finally introduce the method that we will call tree-hypersequent method. In order to do it, we begin by informally explaining what a tree-hypersequent is and we do this by constructing such an object step-by-step. Let us, then, recall, first of all, the simple notion of empty hypersequent: an empty hypersequent is a syntactic object of the following form:

$$
\overbrace{-1-1-}^{n}
$$

which is to say: $n$ slashes which separate $n+1$ dashes. If the order of the dashes is taken on account (as is not standardly done), we can look to this entire structure as a tree-frame in Kripke semantics, where the dashes are meant to be the worlds of the tree-frame and the slashes the relations between worlds in the tree-frame. Following this analogy the dash at distance one in an empty hypersequent denotes a world at distance one in the corresponding tree-frame, a dash at distance two denotes a world a distance two in the corresponding tree-frame, and so on.

In a tree-frame, at every distance, except the first one, we may find $n$ different possible worlds: how can we express this fact in our syntactic object? We separate different dashes with a semi-colon and obtain, in this way, the notion of empty tree-hypersequent. So an example of an empty tree-hypersequent is an object of the following form (the figure on the left):

$$
-/-;-\quad \rightsquigarrow \quad \nwarrow_{0}^{\circ}
$$

which corresponds to a tree-frame (the figure on the right) with a world at distance one related with two different worlds at distance two. Another example of an empty tree-hypersequent is an object of the following form (the figure on the left):

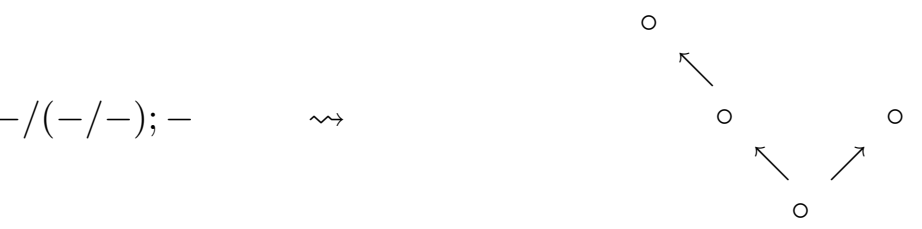

which corresponds to a tree-frame (the figure on the right) with a world at distance one related with two different worlds at distance two, each of which is, in turn, related with another world at distance three. Finally, in order to obtain a tree-hypersequent we fill the dashes with sequents which are objects of the form $M \Rightarrow N$, where $M$ and $N$ are multisets of formulas. 
In the next section we will show how to apply this method in order to obtain calculi for: (i) the basic system $K$, which is valid and complete in all the frames, (ii) the extensions of $K$ which contain one or both the $D$ axiom and the 4 axiom, namely $K D, K 4$ and $K D 4$. We remind the reader that the $D$ axiom has the form: $\square \alpha \rightarrow \diamond \alpha$, and its characteristic frame property is seriality; the 4 axiom, instead, has the form: $\square \alpha \rightarrow \square \square \alpha$, and its characteristic frame property is transitivity. In the third section we will show which rules are admissible in these calculi, in the fourth section we will prove that they are valid and complete in the corresponding Hilbert-style system, and in the fifth section we will finally prove the cut-elimination theorem for all of them.

\section{The Calculi CSK*}

We define the modal propositional language $\mathcal{L}^{\square}$ in the following way:

atoms: $p_{0}, p_{1}, \ldots$

logical constant:

connectives: $\neg, \vee$

The other classic connectives can be defined as usual, as well as the constant $\diamond$ and the formulas of the modal language $\mathcal{L}^{\square}$.

Syntactic Conventions:

$\alpha, \beta, \ldots:$ formulae,

$M, N, \ldots$ finite multisets of formulae,

$\Gamma, \Delta, \ldots:$ sequents (SEQ). The empty sequent $(\Rightarrow)$ is included.

$G, H, \ldots$ : tree-hypersequents (THS).

$\underline{X}, \underline{Y}, \ldots$ : finite multisets of tree-hypersequents (MTHS), $\emptyset$ included.

We point out that for the sake of brevity we will use the following notation: given $\Gamma \equiv M \Rightarrow N$ and $\Pi \equiv P \Rightarrow Q$, we will write $\alpha, \Gamma$ instead of $\alpha, M \Rightarrow N$ and $\Gamma, \alpha$ instead of $M \Rightarrow N, \alpha$, as well as $\Gamma$. $\Pi$ instead of $M, P \Rightarrow N, Q$.

Definition 2.1. The notion of tree-hypersequent is inductively defined in the following way:

- if $\Gamma \in \mathrm{SEQ}$, then $\Gamma \in \mathrm{THS}$,

- if $\Gamma \in \mathrm{SEQ}$ and $\underline{X} \in \mathrm{MTHS}$, then $\Gamma / \underline{X} \in \mathrm{THS}$. 
Given the above definition, an example of tree-hypersequent is the following one:

$$
\Delta /(\Gamma / \Sigma) ;\left(\Gamma_{1} /\left(\Sigma_{1} / \Theta\right) ; \Sigma_{2}\right)
$$

DEFINITION 2.2. The intended interpretation of a tree-hypersequent is:

- $(M \Rightarrow N)^{\tau}:=\bigwedge M \rightarrow \bigvee N$,

- $\left(\Gamma / G_{1} ; \ldots ; G_{n}\right)^{\tau}:=\Gamma^{\tau} \vee \square G_{1}^{\tau} \vee \ldots \vee \square G_{n}^{\tau}$.

Given the above definition, the intended interpretation of the tree-hypersequent of the example above, is:

$$
\Delta^{\tau} \vee \square\left(\Gamma^{\tau} \vee \square \Sigma^{\tau}\right) \vee \square\left(\Gamma_{1}^{\tau} \vee \square\left(\Sigma_{1}^{\tau} \vee \square \Theta^{\tau}\right) \vee \square \Sigma_{3}^{\tau}\right)
$$

In order to display the rules of the calculi, we will use the notation $G[\Gamma]$ (or $G[H])$ to refer to a tree-hypersequent together with a specific occurrence in it of a sequent $\Gamma$ (or a tree-hypersequent $H$ ). You may think, if you like, of $G[$ ] as a "tree-hypersequent with one hole," an object which becomes a real tree-hypersequent whenever a sequent $\Gamma$ (or a tree-hypersequent $H$ ) is appropriately put into the hole.

We can even use the notation $G[\Gamma][[\square \alpha, \Sigma]]$, where $[[\square \alpha, \Sigma]]$ represents all the sequents in $G$ which are successive to $\Gamma$ and contain(ed) the formula $\square \alpha$ on the left side.

The calculus $C S K$ is composed of:

\section{Initial Tree-hypersequents.}

$G[p, \Gamma, p]$

\section{Propositional Rules.}

$$
\begin{array}{cc}
\frac{G[\Gamma, \alpha]}{G[\neg \alpha, \Gamma]} \neg A & \frac{G[\alpha, \Gamma]}{G[\Gamma, \neg \alpha]} \neg K \\
\frac{G[\alpha, \beta, \Gamma]}{G[\alpha \wedge \beta, \Gamma]} \wedge A & \frac{G[\Gamma, \alpha] \quad G[\Gamma, \beta]}{G[\Gamma, \alpha \wedge \beta]} \wedge K
\end{array}
$$

\section{Modal Rules.}

$$
\frac{G\left[\square \alpha, \Gamma /(\alpha, \Sigma / \underline{X}) ; \underline{X}^{\prime}\right]}{G\left[\square \alpha, \Gamma /(\Sigma / \underline{X}) ; \underline{X}^{\prime}\right]} \square A \quad \frac{G[\Gamma / \Rightarrow \alpha ; \underline{X}]}{G[\Gamma, \square \alpha / \underline{X}]} \square K
$$

We underline that the addition of the formula $\square \alpha$ to the left side of the sequent of the premise of the rule $\square A$ only serves to make the rule invertible. 
This is analogous to the repetition of the formula $\forall x(\alpha)$ in the premise of the rule which introduces the universal quantification, in some versions of the sequent calculus of first-order logic.

In order to introduce the cut-rule, we firstly need two new notions:

Definition 2.3. Given two tree-hypersequents, $G[\Gamma]$ and $G^{\prime}\left[\Gamma^{\prime}\right]$ together with an occurrence of a sequent in each, the relation of equivalent position between two of their sequents, in this case $\Gamma$ and $\Gamma^{\prime}, G[\Gamma] \sim G^{\prime}\left[\Gamma^{\prime}\right]$, is defined inductively in the following way:

$-\quad \Gamma \sim \Gamma^{\prime}$

- $\quad \Gamma / \underline{X} \sim \Gamma^{\prime} / \underline{X}^{\prime}$

- $\quad$ If $H[\Gamma] \sim H^{\prime}\left[\Gamma^{\prime}\right]$, then $\Delta / H[\Gamma] ; \underline{X} \sim \Delta^{\prime} / H^{\prime}\left[\Gamma^{\prime}\right] ; \underline{X^{\prime}}$

Intuitively, given two tree-hypersequents, $G[\Gamma]$ and $G^{\prime}\left[\Gamma^{\prime}\right]$ together with an occurrence of a sequent in each, the relation of equivalent position between two of their sequents holds when, by considering $G[\Gamma]$ and $G^{\prime}\left[\Gamma^{\prime}\right]$ as trees, and $\Gamma$ and $\Gamma^{\prime}$ as nodes of the trees, the two nodes have the same height in their respective trees. Consider for example the two tree-hypersequents $G \equiv$ $\Delta /(\Gamma / \Sigma) ;\left(\Gamma_{1} /\left(\Sigma_{1} / \Theta\right) ; \Sigma_{2}\right)$ and $G^{\prime} \equiv \Delta^{\prime} /\left(\Gamma^{\prime} / \Sigma^{\prime}\right) ;\left(\Gamma_{1}^{\prime}\right) ;\left(\Gamma_{2}^{\prime} /\left(\Sigma_{1}^{\prime} / \Theta^{\prime}\right)\right)$. Then $\Gamma$ and $\Gamma^{\prime}$ are in equivalent position, as are $\Gamma$ and $\Gamma_{1}^{\prime}$, or $\Theta$ and $\Theta^{\prime}$.

Definition 2.4. Given two tree-hypersequents $G[\Gamma]$ and $G^{\prime}\left[\Gamma^{\prime}\right]$ together with an occurrence of a sequent in each, such that $G[\Gamma] \sim G^{\prime}\left[\Gamma^{\prime}\right]$, the operation of product, $G[\Gamma] \otimes G^{\prime}\left[\Gamma^{\prime}\right]$, is defined inductively in the following way:

- $\quad \Gamma \otimes \Gamma^{\prime}:=\Gamma \cdot \Gamma^{\prime}$

- $\quad(\Gamma / \underline{X}) \otimes\left(\Gamma^{\prime} / \underline{X}^{\prime}\right):=\Gamma \cdot \Gamma^{\prime} / \underline{X} ; \underline{X^{\prime}}$

- $(\Delta / H[\Gamma] ; \underline{X}) \otimes\left(\Delta^{\prime} / H^{\prime}\left[\Gamma^{\prime}\right] ; \underline{X}^{\prime}\right):=$ $\Delta \cdot \Delta^{\prime} /\left(H[\Gamma] \otimes H^{\prime}\left[\Gamma^{\prime}\right]\right) ; \underline{X} ; \underline{X^{\prime}}$

\section{Cut rule.}

Given two tree-hypersequents $G[\Gamma, \alpha]$ and $G^{\prime}[\alpha, \Pi]$ together with an occurrence of a sequent in each, such that $G[\Gamma, \alpha] \sim G^{\prime}[\alpha, \Pi]$, the cut rule is:

$$
\frac{G[\Gamma, \alpha] \quad G^{\prime}[\alpha, \Pi]}{G \otimes G^{\prime}[\Gamma \cdot \Pi]} c u t_{\alpha}
$$

As the reader can easily see from the above definition, the cut rule should respect two important criteria. The first one says that, given two treehypersequents, we can cut on any two sequents belonging to them provided 
that they are in equivalent position. The second one says that after the cut the two tree-hypersequents should not be randomly mixed but according to the inductive definition of product. We underline that these two criteria are fundamental because they serve to assure that the result of a cut between two tree-hypersequents is still a tree-hypersequent, which is to say the tree shape is kept.

The corresponding rules of axiom $D$ and axiom 4 are, respectively, the following two ones:

$$
\frac{G[\Gamma / \Rightarrow]}{G[\Gamma]} \text { ser. } \quad \frac{G\left[\square \alpha, \Gamma /(\square \alpha, \Sigma / \underline{X}) ; \underline{X}^{\prime}\right]}{G\left[\square \alpha, \Gamma /(\Sigma / \underline{X}) ; \underline{X}^{\prime}\right]} \text { tran. }
$$

Sequent calculi $C S K D, C S K 4$ and $C S K D 4$ can be obtained by adding to the basic sequent calculus $C S K$ one or both the above rules.

In next section we will use the notation $C S K^{*}$ (or, if necessary, $C S K D^{*}$ and $\left.C S K 4^{*}\right)$ to denote the calculus $C S K(C S K D, C S K 4)$ and its extensions.

\section{Admissibility of the Structural Rules}

In this section we will show which structural rules are admissible in calculi $C S K^{*}$. Moreover, in order to show that the two rules of contraction are height-preserving admissible we will show that all the logical and modal rules are height-preserving invertible. The proof of the admissibility of the cut-rule will be shown in the fifth section.

DeFinition 3.1. We associate to each proof $d$ in $C S K^{*}$ a natural number $h(d)$ (height). Intuitively, the height corresponds to the length of the longest branch in a tree-proof $d$, minus one. However we omit the standard inductive definition.

Definition 3.2. $d \vdash^{n} G$ means that $d$ is a proof of $G$ in $C S K^{*}$, with $h(d)$ $\leq n$. We write $\vdash^{\langle n\rangle} G$ for: "there exists a proof $d$ such that $d \vdash^{n} G$."

Definition 3.3. Let $G$ be a tree-hypersequent and $G^{\prime}$ be the result of the application of a certain rule $\mathcal{R}$ on $G$. We say that this rule $\mathcal{R}$ is heightpreserving admissible when:

$$
d \vdash^{n} G \quad \Rightarrow \quad \exists d^{\prime}\left(d^{\prime} \vdash^{n} G^{\prime}\right)
$$

We call a rule, $\mathcal{R}$, which transforms a tree-hypersequent $G$ into a treehypersequent $G^{\prime}$, admissible when:

$$
d \vdash^{n} G \quad \Rightarrow \quad \exists d^{\prime}\left(d^{\prime} \vdash G^{\prime}\right)
$$


Observation 3.4. In the sequent calculus for classical logic, we usually say that a formula of a sequent is principal in a rule when the rule operates on that formula. In a similar way we will call a sequent(s) principal in a tree-hypersequent when a certain rule operates on that sequent(s). In the following proofs of the (height-preserving) admissibility of structural rules and height-preserving invertibility of logical and modal rules, we will consider only these cases where the sequent(s) is (are) principal. All the other cases are dealt with easily, as shown in the two lemmas 3.14 and 3.15 which are proved at the end of the current section.

Lemma 3.5. Tree-hypersequents of the form $G[\alpha, \Gamma, \alpha]$, with $\alpha$ an arbitrary modal formula, are derivable in $C S K^{*}$.

Proof. By straightforward induction on $\alpha$.

LEMMA 3.6. The rule:

$$
\frac{G}{\Rightarrow / G} R N
$$

is height-preserving admissible in $C S K^{*}$.

Proof. By induction on the derivation of the premise.

If $G$ is an initial tree-hypersequent, then $\Rightarrow / G$ is also an initial treehypersequent.

If $G$ is inferred by a logical rule, then the inference is clearly preserved. We will give an example using the logical rule $\neg K$ :

$$
\frac{\langle n-1\rangle G[\alpha, \Gamma]}{\langle n\rangle G[\Gamma, \neg \alpha]} \neg K \quad \rightsquigarrow^{2} \quad \frac{\langle n-1\rangle \Rightarrow / G[\alpha, \Gamma]}{\langle n\rangle \Rightarrow / G[\Gamma, \neg \alpha]} \neg K
$$

If $G$ is inferred by the modal rules, these are clearly preserved. We will give an example using the modal rule $\square K$ :

$$
\begin{aligned}
& \frac{\langle n-1\rangle G[\Gamma / \Rightarrow \alpha ; \underline{X}]}{\langle n\rangle G[\Gamma, \square \alpha / \underline{X}]} \square K \\
& \frac{\langle n-1\rangle \Rightarrow / G[\Gamma / \Rightarrow \alpha ; \underline{X}]}{\langle n\rangle \Rightarrow / G[\Gamma, \square \alpha / \underline{X}]} \square K
\end{aligned}
$$

If, finally, $G$ is inferred by rule ser. or rule tran., these are clearly preserved. We will give an example using the rule ser.:

\footnotetext{
${ }^{2}$ The symbol $\rightsquigarrow$ means: the premise of the right side is concluded by induction hypothesis on the premise of the left side.
} 


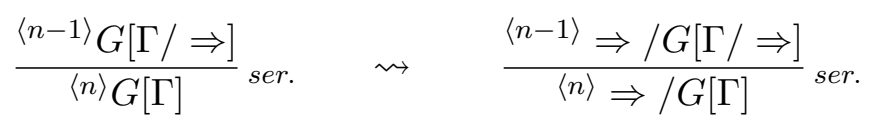

LEMMA 3.7. The rules of weakening:

$$
\frac{G[\Gamma]}{G[\alpha, \Gamma]} W A \quad \frac{G[\Gamma]}{G[\Gamma, \alpha]} W K
$$

are height-preserving admissible in $C S K^{*}$.

Proof. By straightforward induction on the derivation of the premise.

LEMMA 3.8. The rule of external weakening:

$$
\frac{G[\Gamma / \underline{X}]}{G[\Gamma / \underline{X} ; \Gamma]} E W
$$

is height-preserving admissible in $C S K^{*}$.

Proof. By straightforward induction on the derivation of the premise.

LEMma 3.9. The rule of merge:

$$
\frac{G\left[\Delta /\left(\Gamma / \underline{X_{1}}\right) ;\left(\Pi / \underline{X_{2}}\right) ; \underline{Y}\right]}{G\left[\Delta /\left(\Gamma \cdot \Pi / \underline{X_{1}} ; \underline{X_{2}}\right) ; \underline{Y}\right]} \text { merge }
$$

is height-preserving admissible in $C S K^{*}$.

Proof. By induction on the derivation of the premise.

If the premise is an initial tree-hypersequent, then so is the conclusion. If the premise is inferred by a logical rule, this inference is preserved. As the rule of merge has two principal sequents, we should analyze the following two cases: one in which the logical rule has been applied to the sequent $\Gamma$, one in which the logical rule has been applied on the sequent $\Pi$. These two cases are similar; hence we will only sketch the proof for one of them, taking as example the logical rule $\neg K$ :

$$
\begin{aligned}
& \frac{\langle n-1\rangle G\left[\Delta /\left(\alpha, \Gamma / \underline{X_{1}}\right) ;\left(\Pi / \underline{X_{2}}\right) ; \underline{Y}\right]}{\langle n\rangle_{G}\left[\Delta /\left(\Gamma, \neg \alpha / \underline{X_{1}}\right) ;\left(\Pi / \underline{X_{2}}\right) ; \underline{Y}\right]} \neg K \\
& \frac{\langle n-1\rangle_{G}\left[\Delta /\left(\alpha, \Gamma . \Pi / \underline{X_{1}} ; \underline{X_{2}}\right) ; \underline{Y}\right]}{{ }^{\langle n\rangle} G\left[\Delta /\left(\Gamma \cdot \Pi, \neg \alpha / \underline{X_{1}} ; \underline{X_{2}}\right) ; \underline{Y}\right]} \neg K
\end{aligned}
$$

If the premise is inferred by the modal rule $\square K$ (for the rule ser. the treatment is analogous), then as in the case of logical rules, there are two symmetric cases to analyze. We will give an example of just one case: 


$$
\begin{aligned}
& \frac{{ }^{\langle n-1\rangle} G\left[\Delta /\left(\Gamma / \Rightarrow \alpha ; \underline{X}_{1}\right) ;\left(\Pi / \underline{X}_{2}\right) ; \underline{Y}\right]}{\langle n\rangle G\left[\Delta /\left(\Gamma, \square \alpha / \underline{X}_{1}\right) ;\left(\Pi / \underline{X}_{2}\right) ; \underline{Y}\right]} \square K \\
& \frac{\langle n-1\rangle G\left[\Delta /\left(\Gamma \cdot \Pi / \Rightarrow \alpha ; \underline{X}_{1} ; \underline{X}_{2}\right) ; \underline{Y}\right]}{\langle n\rangle G\left[\Delta /\left(\Gamma \cdot \Pi, \square \alpha / \underline{X}_{1} ; \underline{X}_{2}\right) ; \underline{Y}\right]} \square K
\end{aligned}
$$

Finally, in the case where the premise is inferred by the rule tran. (for the rule $\square A$ the treatment is analogous), there are, for a simple combination of principal sequents, two pairs of analogous cases to analyze: on the one hand, tran. applied between $\Delta$ and $\Gamma$, and between $\Delta$ and $\Pi$; on the other hand, tran. applied between $\Gamma$ and $X_{1}$, and between $\Pi$ and $X_{2}$. We will examine one case from each pair:

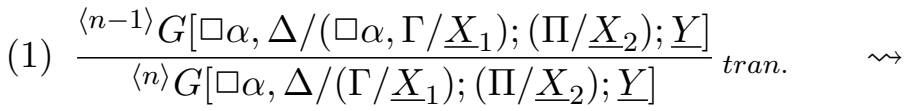

$$
\begin{aligned}
& \frac{\langle n-1\rangle G\left[\square \alpha, \Delta /\left(\square \alpha, \Gamma \cdot \Pi / \underline{X}_{1} ; \underline{X}_{2}\right) ; \underline{Y}\right]}{{ }^{\langle n\rangle} G\left[\square \alpha, \Delta /\left(\Gamma \cdot \Pi / \underline{X}_{1} ; \underline{X}_{2}\right) ; \underline{Y}\right]} \text { tran. } \\
& (2)^{3} \frac{\langle n-1\rangle G\left[\Delta /\left(\square \alpha, \Gamma /\left(\square \alpha, \Sigma / \underline{X}_{1}^{\prime}\right) ; \underline{X}_{1}^{\prime \prime}\right) ;\left(\Pi / \underline{X}_{2}\right) ; \underline{Y}\right]}{{ }^{\langle n\rangle} G\left[\Delta /\left(\square \alpha, \Gamma /\left(\Sigma / \underline{X}_{1}^{\prime}\right) ; \underline{X}_{1}^{\prime \prime}\right) ;\left(\Pi / \underline{X}_{2}\right) ; \underline{Y}\right]} \operatorname{tran} . ~ \rightsquigarrow \\
& \frac{\langle n-1\rangle G\left[\Delta /\left(\square \alpha, \Gamma \cdot \Pi /\left(\square \alpha, \Sigma / \underline{X}_{1}^{\prime}\right) ; \underline{X}_{1}^{\prime \prime} ; \underline{X}_{2}\right) ; \underline{Y}\right]}{{ }^{\langle n\rangle} G\left[\Delta /\left(\square \alpha, \Gamma \cdot \Pi /\left(\Sigma / \underline{X}_{1}^{\prime}\right) ; \underline{X}_{1}^{\prime \prime} ; \underline{X}_{2}\right) ; \underline{Y}\right]} \text { tran. }
\end{aligned}
$$

LEMMA 3.10. The following rule:

$$
\frac{G\left[\Gamma /(\Sigma / \underline{X}) ; \underline{X}^{\prime}\right]}{G\left[\Gamma /(\Rightarrow / \Sigma / \underline{X}) ; \underline{X}^{\prime}\right]} \text { tran.2. }
$$

is admissible in those calculi which contain the rule tran.

Proof. By induction on the derivation of the premise. The cases where the premise is an initial tree-hypersequent or is preceded by a logical rule are trivial. We analyze the cases in which the last applied rule is one of the modal rules or is the rule ser. or is the rule tran.

$[\square K]$ (for the rule ser. the treatment is analogous):

$$
\begin{aligned}
& \frac{\langle n-1\rangle G[\Gamma / \Rightarrow \alpha ;(\Sigma / \underline{X}) ; \underline{Y}]}{\langle n\rangle G[\Gamma, \square \alpha /(\Sigma / \underline{X}) ; \underline{Y}]} \square K \\
& \frac{G[\Gamma / \Rightarrow \alpha ;(\Rightarrow / \Sigma / \underline{X}) ; \underline{Y}]}{G[\Gamma, \square \alpha /(\Rightarrow / \Sigma / \underline{X}) ; \underline{Y}]} \square K
\end{aligned}
$$

\footnotetext{
${ }^{3}$ We take $\underline{X}_{1} \equiv\left(\Sigma / \underline{X}_{1}^{\prime}\right) ; \underline{X}_{1}^{\prime \prime}$
} 


$$
\begin{aligned}
& {[\square A:] \frac{\langle n-1\rangle G[\square \alpha, \Gamma /(\alpha, \Sigma / \underline{X}) ; \underline{Y}]}{{ }^{\langle n\rangle} G[\square \alpha, \Gamma /(\Sigma / \underline{X}) ; \underline{Y}]} \square A \quad \rightsquigarrow} \\
& \frac{G[\square \alpha, \Gamma /(\Rightarrow / \alpha, \Sigma / \underline{X}) ; \underline{Y}]}{\frac{G[\square \alpha, \Gamma /(\square \alpha \Rightarrow / \alpha, \Sigma / \underline{X}) ; \underline{Y}]}{G[\square \alpha, \Gamma /(\square \alpha \Rightarrow / \Sigma / \underline{X}) ; \underline{Y}]} \operatorname{tran} .} A A \\
& {\left[\operatorname{tran} .: \frac{\left\langle{ }^{-1\rangle} G[\square \alpha, \Gamma /(\square \alpha, \Sigma / \underline{X}) ; \underline{Y}]\right.}{\langle n\rangle G[\square \alpha, \Gamma /(\Sigma / \underline{X}) ; \underline{Y}]} \operatorname{tran} . \quad \rightsquigarrow\right.} \\
& \frac{G[\square \alpha, \Gamma /(\Rightarrow / \square \alpha, \Sigma / \underline{X}) ; \underline{Y}]}{\frac{G[\square \alpha, \Gamma /(\square \alpha \Rightarrow / \square \alpha, \Sigma / \underline{X}) ; \underline{Y}]}{G[\square \alpha, \Gamma /(\square \alpha \Rightarrow / \Sigma / \underline{X}) ; \underline{Y}]} \operatorname{tran} .} A A
\end{aligned}
$$

Lemma 3.11. All the logical and modal rules of CSK $K^{*}$ are height-preserving invertible.

Proof. The proof proceeds by induction on the derivation of the premise of the rule considered. The cases of logical rules are dealt with in the classical way. The only differences - the fact that we are dealing with treehypersequents, and the cases where the rule before the logical rule is $\square A$ or $\square K$ or ser. or tran. - are dealt with easily.

The rule $(\square A)$ is trivially height-preserving invertible since the premise is concluded by weakening from the conclusion, and weakening is heightpreserving admissible.

We show in detail the invertibility of the rule $(\square K)$. If $G[\Gamma, \square \alpha / \underline{X}]$ is an initial tree-hypersequent, then so is $G[\Gamma / \Rightarrow \alpha ; \underline{X}]$. If $G[\Gamma, \square \alpha / \underline{X}]$ is preceded by a logical rule $\mathcal{R}$, we apply the inductive hypothesis on the premise(s), $G\left[\Gamma^{\prime}, \square \alpha / \underline{X}\right]\left(G\left[\Gamma^{\prime \prime}, \square \alpha / \underline{X}\right]\right)$ and we obtain derivation(s), of height $n-1$, of $G\left[\Gamma^{\prime} / \Rightarrow \alpha ; \underline{X}\right]\left(G\left[\Gamma^{\prime \prime} / \Rightarrow \alpha ; \underline{X}\right]\right)$. By applying the rule $\mathcal{R}$, we obtain a derivation of height $n$ of $G[\Gamma / \Rightarrow \alpha ; \underline{X}]$. If $G[\Gamma, \square \alpha / \underline{X}]$ is of the form $G\left[\square \beta, \Gamma, \square \alpha /\left(\Sigma / \underline{X}^{\prime}\right) ; \underline{X}^{\prime \prime}\right]$ and is concluded by the modal rule $\square A$, we apply the inductive hypothesis on $G\left[\square \beta, \Gamma, \square \alpha /\left(\beta, \Sigma / \underline{X}^{\prime}\right) ; \underline{X}^{\prime \prime}\right]$ and we obtain a derivation of height $n-1$ of $G\left[\square \beta, \Gamma / \Rightarrow \alpha ;\left(\beta, \Sigma / \underline{X}^{\prime}\right) ; \underline{X}^{\prime \prime}\right]$. By applying the rule $\square A$, we obtain a derivation of height $n$ of $G\left[\square \beta, \Gamma / \Rightarrow \alpha ;\left(\Sigma / \underline{X}^{\prime}\right) ; \underline{X}^{\prime \prime}\right]$. If $G[\Gamma, \square \alpha / \underline{X}]$ is concluded by the rule ser. or tran. or by the modal rule $\square K$ in which $\square \alpha$ is not the principal formula, these cases are analogous to the one of $\square A$. Finally, if $G[\Gamma, \square \alpha / \underline{X}]$ is preceded by the modal rule $\square K$ and $\square \alpha$ is a principal formula, the premise of the last step gives the conclusion. 
LEMMA 3.12. The rules of contraction:

$$
\frac{G[\alpha, \alpha, \Gamma]}{G[\alpha, \Gamma]} C A \quad \frac{G[\Gamma, \alpha, \alpha]}{G[\Gamma, \alpha]} C K
$$

are height-preserving admissible in $C S K^{*}$.

Proof. By induction on the derivation of the premise $G[\Gamma, \alpha, \alpha]$. We only analyze the case of the rule $C K$. The case of the rule $C A$ is symmetric.

If $G[\Gamma, \alpha, \alpha]$ is an initial tree-hypersequent, so is $G[\Gamma, \alpha]$.

If $G[\Gamma, \alpha, \alpha]$ is preceded by a rule $\mathcal{R}$ which does not have any of the two occurrences of the formula $\alpha$ as principal, we apply the inductive hypothesis on the premise(s) $G^{\prime}\left[\Gamma^{\prime}, \alpha, \alpha\right]\left(G^{\prime \prime}\left[\Gamma^{\prime \prime}, \alpha, \alpha\right]\right)$, obtaining derivation(s) of height $n-1$ of $G^{\prime}\left[\Gamma^{\prime}, \alpha\right]\left(G^{\prime \prime}\left[\Gamma^{\prime \prime}, \alpha\right]\right)$. By applying the rule $\mathcal{R}$ we obtain a derivation of height $n$ of $G[\Gamma, \alpha]$

$G[\Gamma, \alpha, \alpha]$ is preceded by a logical or modal rule and one of the two occurrences of the formula $\alpha$ is principal. Hence the rule which concludes $G[\Gamma, \alpha, \alpha]$ is a $K$-rule and we have to analyze the following three cases: $\neg K$, $\wedge K, \square K$.

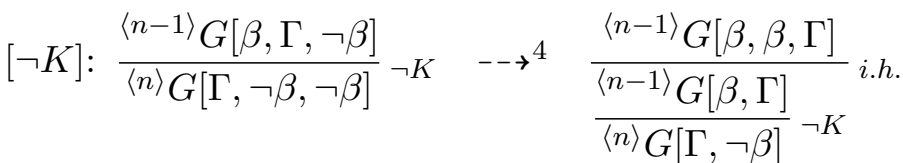

$$
\begin{aligned}
& {[\wedge K]: \frac{\langle n-1\rangle G[\Gamma, \beta, \beta \wedge \gamma] \quad\langle n-1\rangle G[\Gamma, \gamma, \beta \wedge \gamma]}{\langle n\rangle G[\Gamma, \beta \wedge \gamma, \beta \wedge \gamma]} \wedge K \quad \rightarrow} \\
& \frac{\frac{\langle n-1\rangle G[\Gamma, \beta, \beta]}{\langle n-1\rangle G[\Gamma, \beta]} i . h \quad \frac{\langle n-1\rangle G[\Gamma, \gamma, \gamma]}{\langle n-1\rangle G[\Gamma, \gamma]} i . h .}{\langle n\rangle G[\Gamma, \beta \wedge \gamma]} \wedge K
\end{aligned}
$$

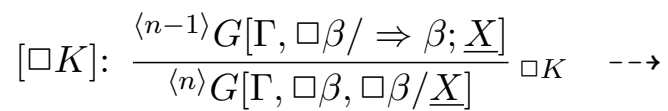

$$
\begin{aligned}
& \frac{{ }^{\langle n-1\rangle} G[\Gamma / \Rightarrow \beta ; \Rightarrow \beta ; \underline{X}]}{\frac{{ }^{\langle n-1\rangle} G[\Gamma / \Rightarrow \beta, \beta ; \underline{X}]}{{ }^{\left.{ }^{n}-1\right\rangle} G[\Gamma / \Rightarrow \beta ; \underline{X}]}} \text { merge }
\end{aligned}
$$

LEMma 3.13. The rule of external contraction:

$$
\frac{G\left[\Gamma /\left(\Sigma / \underline{X_{1}}\right) ;\left(\Sigma / \underline{X_{2}}\right) ; \underline{Y}\right]}{G\left[\Gamma /\left(\Sigma / \underline{X_{1}} ; \underline{X_{2}}\right) ; \underline{Y}\right]} E C
$$

is height-preserving admissible 
ProOF. $^{5}$

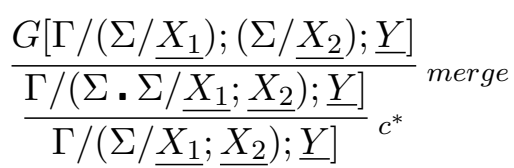

Lemma 3.14. Let $G[H]$ be any tree-hypersequent and $G^{*}[H]$ the result of the application of one of the structural rules - classical and external weakening, merge, tran2. and classical contraction - on $G[H]$. If for a rule $\mathcal{R}$ we have:

$$
\frac{G\left[H^{\prime}\right]}{G[H]} \mathcal{R}
$$

then it holds that:

$$
\frac{G^{*}\left[H^{\prime}\right]}{G^{*}[H]} \mathcal{R}
$$

Proof. By induction on the form of the tree-hypersequent $G[H]$.

Lemma 3.15. Let $G[H]$ be any tree-hypersequent and $G\left[H^{\prime}\right]$ the result of the application of one of the logical rules or the rule $\square K$ on $G[H]$. If for a rule $\mathcal{R}$ we have:

$$
\frac{G^{*}\left[H^{\prime}\right]}{G\left[H^{\prime}\right]} \mathcal{R}
$$

then it holds that:

$$
\frac{G^{*}[H]}{G[H]} \mathcal{R}
$$

Proof. By induction on the form of the tree-hypersequent $G\left[H^{\prime}\right]$.

\section{The adequateness of the calculi}

In this section we briefly prove that our calculi $C S K^{*}$ prove exactly the same formulas as their corresponding Hilbert-style systems, that from now on, we will indicate with the notation $K^{*}$.

Theorem 4.1. [i] If $\vdash \alpha$ in $K^{*}$, then $\vdash \Rightarrow \alpha$ in $C S K^{*}$.

[ii] If $\vdash G$ in $C S K^{*}$, then $\vdash(G)^{\tau}$ in $K^{*}$.

\footnotetext{
${ }^{5}$ In the last inference of the proof, if the proof is read bottom up, we use the rule of negation twice in a role. From now on we indicate the repeated running applications of a same rule on a tree-hypersequent, by writing the rule with the symbol ${ }^{*}$ as index.
} 
Proof. By induction on the height of proofs in $K^{*}$ and $C S K^{*}$, respectively. As concerns [ii], we omit the proof which is easy but quite tedious. However the technique to develop such proof consists of the following two steps: first of all, the sequent(s) affected by the rule should be isolated and the corresponding implication proved, then the implication should be transported up all along the tree so that, by modus ponens, the desired result is immediately achieved. In order to further acquaint the reader with the calculi $C S K^{*}$ we verify [i]. The classical axioms and the modus ponens rule are proved as usual, we just present the proof of the distribution axiom, axiom $D$, axiom 4 and the necessity rule.

$$
\begin{aligned}
& C S K^{*} \vdash \Rightarrow(\alpha \rightarrow \beta) \rightarrow \square \alpha \rightarrow \square \beta \\
& \frac{\square(\alpha \rightarrow \beta) \Rightarrow / \alpha \Rightarrow \alpha \quad \square \alpha \Rightarrow / \beta \Rightarrow \beta}{\square(\alpha \rightarrow \beta), \square \alpha \Rightarrow / \alpha \rightarrow \beta, \alpha \Rightarrow \beta} \rightarrow A \\
& \overline{\square(\alpha \rightarrow \beta), \square \alpha \Rightarrow / \alpha \Rightarrow \beta} \square A \\
& \frac{\square(\alpha \rightarrow \beta), \square \alpha \Rightarrow / \Rightarrow \beta}{\square(\alpha \rightarrow \beta), \square \alpha \Rightarrow \square \beta} \square A \\
& \overline{\square(\alpha \rightarrow \beta) \Rightarrow \square \alpha \rightarrow \square \beta} \rightarrow K=K \\
& \overline{\Rightarrow \square(\alpha \rightarrow \beta) \rightarrow \square \alpha \rightarrow \square \beta} \rightarrow K \\
& C S K D^{*} \vdash \Rightarrow \square \alpha \rightarrow \neg \square \neg \alpha \\
& \begin{array}{l}
\frac{\square \alpha, \square \neg \alpha \Rightarrow / \alpha \Rightarrow \alpha}{\square \alpha, \square \neg \alpha \Rightarrow / \neg \alpha, \alpha \Rightarrow} \\
\frac{\square \alpha, \square \neg \alpha \Rightarrow / \alpha \Rightarrow A}{\square \alpha, \square \neg \alpha \Rightarrow / \Rightarrow} \square A \\
\frac{\square \alpha, \square \neg \alpha \Rightarrow}{\square \alpha \Rightarrow \neg \square} \text { ser. } \\
\frac{\square \alpha \alpha}{\Rightarrow \alpha \alpha \rightarrow \neg \neg} \rightarrow K
\end{array} \\
& C S K 4^{*} \vdash \Rightarrow \square \rightarrow \square \square \alpha \\
& \begin{array}{l}
\frac{\square \alpha / \square \alpha \Rightarrow / \alpha \Rightarrow \alpha}{\square \alpha \Rightarrow / \square \alpha \Rightarrow / \Rightarrow \alpha} \square A \\
\frac{\square \alpha \Rightarrow / \square \alpha \Rightarrow \square \alpha}{\square \alpha \Rightarrow / \Rightarrow \square \alpha} \text { tran. } \\
\frac{\square \alpha \Rightarrow \square \square \alpha}{\Rightarrow \square \alpha \rightarrow \square \square \alpha} \rightarrow K
\end{array}
\end{aligned}
$$

if $C S K^{*} \vdash \Rightarrow \alpha$, then $C S K^{*} \vdash \Rightarrow \square \alpha$

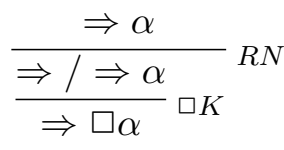




\section{Cut-elimination Theorem for CSK*}

In this section we prove that the cut-rule is admissible in calculi $C S K^{*}$. In order to prove such a theorem we firstly have to show the following lemma.

LEMma 5.1. Given three tree-hypersequents together with a displayed occurrence of a sequent $\Gamma, I[\Gamma], J[\Gamma]$ and $H[\Gamma]$ such that $I[\Gamma] \sim J[\Gamma] \sim H[\Gamma]$, if there is a rule $\mathcal{R}$ such that:

$$
\frac{J[\Gamma]}{I[\Gamma]} \mathcal{R}
$$

then, for every $\Delta$ it holds:

$$
\frac{J \otimes H[\Delta]}{I \otimes H[\Delta]} \mathcal{R}
$$

Proof. By induction on the form of the tree-hypersequents $I[\Gamma], J[\Gamma]$ and $H[\Gamma]$.

Now we can prove that the cut-rule is admissible in the calculi $C S K^{*}$.

Theorem 5.2. Let $G[\Gamma, \alpha]$ and $G^{\prime}[\alpha, \Pi]$ be such that $G[\Gamma, \alpha] \sim G^{\prime}[\alpha, \Pi]$. If:

$$
\begin{array}{cc}
\vdots & \vdots \\
d_{1} & d_{2} \\
G[\Gamma, \alpha] & G[\alpha, \Pi] \\
G^{\prime}[\Gamma \cdot \Pi] & \text { cut }_{\alpha}
\end{array}
$$

and $d_{1}$ and $d_{2}$ do not contain any other application of the cut rule, then we can construct a proof of $G \otimes G^{\prime}[\Gamma \cdot \Pi]$ without any application of cut rule.

Proof. The proof is developed by induction on the complexity of the cut formula, which is the number $(\geq 0)$ of the occurrences of logical symbols in cut formula $\alpha$, with subinduction on the sum of the heights of the derivations of the premises of cut. We will distinguish cases by the last rule applied on the left premise.

Case 1. $G[\Gamma, \alpha]$ is an initial tree-hypersequent. Then either the conclusion is also a tree-hypersequent or the cut can be replaced by various applications of the classical and external weakening rules on $G^{\prime}[\alpha, \Pi]$.

Case 2. $G[\Gamma, \alpha]$ is inferred by a rule $\mathcal{R}$ in which $\alpha$ is not principal. This case can be standardly solved, by induction on the sum of the heights of the derivations of the premises of cut. Indeed there is no rule which is able to change the position of the sequent where we cut, and, on the other hand, the definition of product assures us that the structure of the treehypersequent stay unchanged, therefore no problem arises. However, for the 
sake of clarity, let us make some examples. More particularly we will analyze those significant cases where the rule $\mathcal{R}$ has been applied on the sequent $\Gamma, \alpha$. The others can be dealt with analogously, thanks to the lemma 5.1. Let us then suppose that the rule before $G[\Gamma, \alpha]$ is the rule $\square K$ (the case where $\mathcal{R}$ is the rule ser. is analogous) applied on the sequent $\Gamma, \alpha$ and without $\alpha$ as principal formula. We have:

$$
\frac{\frac{G[\Gamma, \alpha / \Rightarrow \beta ; \underline{X}]}{G[\Gamma, \alpha, \square \beta / \underline{X}]} \square K \quad G^{\prime}[\alpha, \Pi / \underline{Y}]}{G \otimes G^{\prime}[\Gamma \cdot \Pi, \square \beta / \underline{X} ; \underline{Y}]} c_{\alpha}
$$

We reduce to:

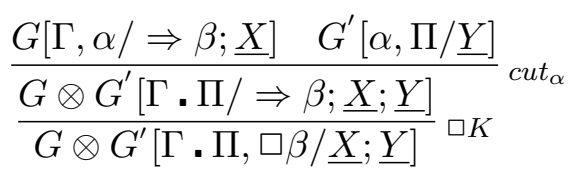

Let us suppose that the rule before $G[\Gamma, \alpha]$ is the rule $\square A$ (the case where $\mathcal{R}$ is the rule tran. is analogous) applied between the sequent $\Gamma, \alpha$ and the sequent successive to it, and without $\alpha$ as principal formula. We have:

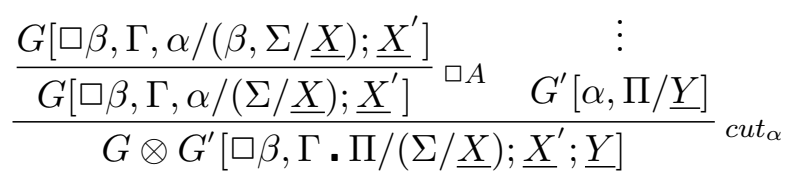

We reduce to:

$$
\frac{G\left[\square \beta, \Gamma, \alpha /(\beta, \Sigma / \underline{X}) ; \underline{X}^{\prime}\right] \quad G^{\prime}[\alpha, \Pi / \underline{Y}]}{G \otimes G^{\prime}\left[\square \beta, \Gamma \cdot \Pi /(\beta, \Sigma / \underline{X}) ; \underline{X^{\prime}} ; \underline{Y}\right]} c u t \alpha_{\alpha}
$$

Let us finally suppose that the rule before $G[\Gamma, \alpha]$ is the rule $\square A$ (the case where $\mathcal{R}$ is the rule tran. is analogous) applied between the sequent $\Gamma, \alpha$ and the sequent which precedes it, and without $\alpha$ as principal formula. We have:

$$
\frac{\frac{G\left[\square \beta, \Delta /(\beta, \Gamma, \alpha / \underline{X}) ; \underline{X}^{\prime}\right]}{G\left[\square \beta, \Delta /(\Gamma, \alpha / \underline{X}) ; \underline{X}^{\prime}\right]} \square A \quad G^{\prime}\left[\Lambda /(\alpha, \Pi / \underline{Y}) ; \underline{Y}^{\prime}\right]}{G \otimes G^{\prime}\left[\square \beta, \Delta . \Lambda /(\Gamma \cdot \Pi / \underline{X} ; \underline{Y}) ; \underline{X}^{\prime} ; \underline{Y}^{\prime}\right]} c u t_{\alpha}
$$

We reduce to:

$$
\frac{G\left[\square \beta, \Delta /(\beta, \Gamma, \alpha / \underline{X}) ; \underline{X}^{\prime}\right] \quad G^{\prime}\left[\Lambda /(\alpha, \Pi / \underline{Y}) ; \underline{Y^{\prime}}\right]}{G \otimes G^{\prime}\left[\square \beta, \Delta \cdot \Lambda /(\beta, \Gamma \cdot \Pi / \underline{X} ; \underline{Y}) ; \underline{X}^{\prime} ; \underline{Y}^{\prime}\right]} c u t_{\alpha}
$$


Case 3. $G[\Gamma, \alpha]$ is inferred by a rule $\mathcal{R}$ in which $\alpha$ is principal. We distinguish two subcases: in one subcase $\mathcal{R}$ is a logical rule, in the other $\mathcal{R}$ is a modal rule.

Case 3.1. We suppose, as example, that the rule before $G[\Gamma, \alpha]$ is $\neg K$, we have:

$$
\frac{\frac{G[\beta, \Gamma]}{G[\Gamma, \neg \beta]} \neg K \quad G^{\prime}[\neg \beta, \Pi]}{G \otimes G^{\prime}[\Gamma \cdot \Pi]} c u t_{\neg \beta}
$$

By applying lemma 3.11 on $G^{\prime}[\neg \beta, \Pi]$, we obtain $G^{\prime}[\Pi, \beta]$. We replace the previous cut with the following one which is eliminable by induction on the complexity of the cut formula:

$$
\frac{G^{\prime}[\Pi, \beta] \quad G[\beta, \Gamma]}{G \otimes G^{\prime}[\Gamma \cdot \Pi]} \text { cut }_{\beta}
$$

Case 3.2. $\mathcal{R}$ is $\square K$ and $\alpha \equiv \square \beta$. We have the following situation:

$$
\frac{\frac{G[\Gamma / \Rightarrow \beta ; \underline{X}]}{G[\Gamma, \square \beta / \underline{X}]} \square K \quad G^{\prime}[\square \beta, \Pi]}{G \otimes G^{\prime}[\Gamma \cdot \Pi / \underline{X}]} c u t_{\square \beta}
$$

We have to consider the last rule $\mathcal{R}^{\prime}$ of $d_{2}$. If there is no rule $\mathcal{R}^{\prime}$ which introduces $G^{\prime}[\square \beta, \Pi]$ because $G^{\prime}[\square \beta, \Pi]$ is an initial tree-hypersequent, then we can solve the case as in 1 . If $\mathcal{R}^{\prime}$ is a rule in which $\square \beta$ is not principal, we solve the case as in 2 . The only problematic cases are those cases where $\mathcal{R}^{\prime}$ is $\square A$ or tran. We analyze them both.

$\square A$ :

$$
\frac{\frac{G[\Gamma / \Rightarrow \beta ; \underline{X}]}{G[\Gamma, \square \beta / \underline{X}]} \square K \quad \frac{G^{\prime}\left[\square \beta, \Pi /(\beta, \Phi / \underline{Y}) ; \underline{Y}^{\prime}\right]}{G^{\prime}\left[\square \beta, \Pi /(\Phi / \underline{Y}) ; \underline{Y}^{\prime}\right]} \square A}{G \otimes G^{\prime}\left[\Gamma \cdot \Pi / \underline{X} ;(\Phi / \underline{Y}) ; \underline{Y}^{\prime}\right]} c u t_{\square \beta}
$$

We reduce to:

$$
\begin{gathered}
\frac{G[\Gamma, \square \beta / \underline{X}] \quad G^{\prime}\left[\square \beta, \Pi /(\beta, \Phi / \underline{Y}) ; \underline{Y}^{\prime}\right]}{G \otimes G^{\prime}\left[\Gamma \cdot \Pi / \underline{X} ;(\beta, \Phi / \underline{Y}) ; \underline{Y}^{\prime}\right]} c u t_{\square \beta} \\
\frac{G[\Gamma / \Rightarrow \beta ; \underline{X}] \quad G \otimes G^{\prime}\left[\Gamma \cdot \Pi / \underline{X} ;(\beta, \Phi / \underline{Y}) ; \underline{\left.Y^{\prime}\right]}\right.}{\frac{G \otimes G \otimes G^{\prime}\left[\Gamma \cdot \Gamma \cdot \Pi / \underline{X} ; \underline{X} ;(\Phi / \underline{Y}) ; \underline{Y^{\prime}}\right]}{G \otimes G^{\prime}\left[\Gamma \cdot \Pi / \underline{X} ;(\Phi / \underline{Y}) ; \underline{Y}^{\prime}\right]} C^{*}}
\end{gathered}
$$


where the first cut is eliminable by induction on the sum of the heights of the derivations of the premises of cut and the second cut is eliminable by induction on the complexity of cut formula.

tran.:

$$
\frac{\frac{G[\Gamma / \Rightarrow \beta ; \underline{X}]}{G[\Gamma, \square \beta / \underline{X}]} \square K \quad \frac{G^{\prime}\left[\square \beta, \Pi /(\square \beta, \Phi / \underline{Y}) ; \underline{Y}^{\prime}\right]}{G^{\prime}\left[\square \beta, \Pi /(\Phi / \underline{Y}) ; \underline{Y}^{\prime}\right]} \operatorname{tran.}}{G \otimes G^{\prime}\left[\Gamma \cdot \Pi / \underline{X} ;(\Phi / \underline{Y}) ; \underline{Y}^{\prime}\right]} C u t_{\square \beta}
$$

In order to solve this case, we must check what can have introduced the tree-hypersequent $G^{\prime}\left[\square \beta, \Pi /(\square \beta, \Phi / \underline{Y}) ; \underline{Y}^{\prime}\right]$. More particularly we go up the derivation until either a rule applies to a formula different from the $\square \beta$ 's or a rule different from tran. applies to some of the $\square \beta$ 's. This way we have the following situation:

$$
\diamond: \quad G^{\prime}[\square \beta, \Pi][[\square \beta, \Psi]]^{6}
$$

We then analyze each of the rules which can have inferred the tree-hypersequent $\diamond$ :

- $\diamond$ is an axiom. Then, as $\square \beta$ cannot be principal, even the conclusion of the cut is an axiom and the case is solved.

- $\diamond$ has been inferred by a rule $\mathcal{R}^{\prime \prime}$ which does not have any $\square \beta$ as a principal formula. In this case the technique consists of: firstly, permuting the rule $\mathcal{R}^{\prime \prime}$ and the $n$ applications of the rule tran., and, secondly, operating as in case 2 .

- $\diamond$ has been inferred by a rule $\mathcal{R}^{\prime \prime}$ which has $\square \beta$ as principal formula. $\mathcal{R}^{\prime \prime}$ can only be the rule $\square A$. We still have to distinguish two others possibilities. (1) the rule $\square A$ has been applied to one of the sequents which follow the sequent $[\square \beta, \Pi]$. Hence we have the following situation:

$$
\frac{G[\Gamma / \Rightarrow \beta ; \underline{X}]}{\frac{G^{\prime}[\square \beta, \Pi]\left[\left[\square \beta, \Psi /(\beta, \Xi / \underline{Z}) ; \underline{Z^{\prime}}\right]\right]}{G^{\prime}[\square \beta, \Pi]\left[\left[\square \beta, \Psi /(\Xi / \underline{Z}) ; \underline{Z^{\prime}}\right]\right]}}
$$

We proceed within the following three steps:

\footnotetext{
${ }^{6}$ For the sake of brevity, we omit to write: $/\left(\Phi / \underline{Y}^{\prime}\right) ; \underline{Y}^{\prime \prime}$
} 
(i) we apply the rule $\square A$ and the $n$ applications of the rule tran. in a reverse order and so we obtain the tree-hypersequent:

$$
G^{\prime}[\square \beta, \Pi]\left[\left[\Psi /(\beta, \Xi / \underline{Z}) ; \underline{Z}^{\prime}\right]\right]
$$

(ii) we apply the rule $\operatorname{tran} 2$. to the tree-hypersequent $G[\Gamma / \Rightarrow \beta ; \underline{X}]$ a number of time sufficient to get $\Rightarrow \beta$ in an equivalent position with the sequence $\beta, \Xi$ of the tree-hypersequent $G^{\prime}[\square \beta, \Pi]\left[\left[\Psi /(\beta, \Xi / \underline{Z}) ; \underline{Z^{\prime}}\right]\right]$. This way we obtain a tree-hypersequent where $\Rightarrow \beta$ is no longer after $\Gamma$, but $n$ empty sequences after. Let us note this as: $G[\Gamma / \underline{X}][\Rightarrow \beta]$.

(iii) We are now able to apply two cuts: the first eliminable by induction on the sum of the heights, the second by induction on the complexity of the cut formula.

$$
\begin{gathered}
\frac{G[\Gamma, \square \beta / \underline{X}] \quad G^{\prime}[\square \beta, \Pi]\left[\left[\Psi /(\beta, \Xi / \underline{Z}) ; \underline{Z}^{\prime}\right]\right]}{G \otimes G^{\prime}[\Gamma \cdot \Pi / \underline{X}]\left[\left[\Psi /(\beta, \Xi / \underline{Z}) ; \underline{Z^{\prime}}\right]\right]} c u t_{\square \beta} \\
\frac{G[\Gamma / \underline{X}][\Rightarrow \beta] \quad G \otimes G^{\prime}[\Gamma \cdot \Pi / \underline{X}]\left[\left[\Psi /(\beta, \Xi / \underline{Z}) ; \underline{Z^{\prime}}\right]\right]}{G \otimes G \otimes G^{\prime}[\Gamma \cdot \Gamma \cdot \Pi / \underline{X} ; \underline{X}]\left[\left[\Psi /(\Xi / \underline{Z}) ; \underline{Z^{\prime}}\right]\right]} \\
\frac{G \otimes t_{\beta}}{G \otimes} C^{\prime}[\Gamma \cdot \Pi / \underline{X}]\left[\left[\Psi /(\Xi / \underline{Z}) ; \underline{Z^{\prime}}\right]\right]
\end{gathered}
$$

(2) The rule $\square A$, with $\square \beta$ principal formula, has been applied on the sequent $[\square \beta, \Pi]$. In this case we apply, as before, the rule $\square A$ and the $n$ applications of the rule tran. in a reverse order, and we proceed as at the beginning of this case.

\section{Conclusions and Further Work}

In this paper we have presented the tree-hypersequent method applied to the systems $K, K D, K 4$ and $K D 4$. Through the several sections we were given a chance to observe the advantages it has: it satisfies the subformula property, its rules are invertible and they fit the criteria required for a good sequent calculus, all the structural rules can be shown to be admissible, the contraction rules included. Moreover all the proof, as the calculi, are purely syntactic. Therefore the tree-hypersequents calculi seem to enjoy the qualities for being defined as good. Given this situation, two interesting questions seem to arise naturally: is it possible to obtain other results within the tree-hypersequent method? Is it possible to apply the tree-hypersequent method in order to obtain calculi for other systems of modal logic? Let us answer both of them, following the order. 
As concerns the first hunch, it seems possible to prove two flavored results. As already remarked in [2], the tree shape of the hypersequents should help proving the interpolation theorem. Moreover it does not seem a hard work to adapt the technique introduced by Negri in order to prove the decidability theorem in a purely syntactic way (decidability through semantics has already been established in [2]).

As concerns the second question, in the light of what we have already suggested in [10] and what has been analyzed in details in [2], we can claim that the method of tree-hypersequents can be successfully applied to axioms $B$ and $T$ too (as concerns axiom 5, see again [2]). Moreover, as we have shown in [9], the method can be quite naturally modified in order to get a very simple sequent calculus for modal logic $S 5$. Finally it also seems reasonable to apply the method to obtain a sequent calculus for the modal logic of provability $G L$, in a way similar to that employed by Negri.

On the other hand, we have still to investigate the following two questions: the application of the tree-hypersequents method to temporal logics (which seems quite complicated because of the tree shape of our syntactic objects), and a comparison between the tree-hypersequents method and the tableaux systems one (which seems quite natural).

Acknowledgements. I wish to thank Pierluigi Minari for his constant interest in this work and the substantial help with the notation used in the calculi; Brian Hill for the valuable discussions, comments and suggestions that he has dedicated to this paper.

\section{References}

[1] Avron, A., 'The method of hypersequents in the proof theory of propositional nonclassical logic', in C. Steinhorn et J. Strauss W. Hodges, M. Hyland (eds.), Logic: from foundations to applications, Oxford University Press., 1996.

[2] Brünnler, T., 'Deep sequent systems for modal logic', Advances in Modal Logic AiML, 6: 107-119, 2006.

[3] Goble, L., 'Gentzen systems for modal logic', Notre Dame Journal of Formal Logic, 15: 455-461, 1974.

[4] IndrzejCZAK, A., 'Generalised sequent calculus for propositional modal logics', Logica Trianguli, 1: 15-31, 1997.

[5] Kashima, R., 'Cut-free sequent calculi for some tense logics', Studia Logica, 53: 119$135,1994$.

[6] Matsumoto, M., K. eт Ohnishi, 'Gentzen method in modal calculi', Osaka Mathematical Journal, 9 and 11:115-120, 1959.

[7] Mints, G., 'Indexed systems of sequents and cut-elimination', Journal of Philosophical Logic, 26: 671-696, 1997. 
The Method of Tree-hypersequents for Modal Propositional Logic.

[8] Negri, S., 'Proof analysis in modal logic', Journal of Philosophical Logic, 34, 2005.

[9] Poggiolesi, F., 'A cut-free simple sequent calculus for modal logic s5', Submitted to the Review of Symbolic Logic, June 2007.

[10] Poggiolesi, F., 'Sequent calculus for modal logic', Logic Colloqium, 2006.

[11] SAmbin, G., and S. VAlentini, 'The modal logic of provability. the sequential approach', Journal of Philosophical Logic, 11:311-342, 1982.

[12] Wansing, H., 'Sequent calculi for normal modal propositional logics', Journal of Logic and Computation, 4: 125-142, 1994.

Francesca Poggiolesi

Department of Philosophy,

University of Florence,

IHPST,

University of Paris 1,

Via Bolognese 52,

50139, Florence, Italy.

francesca.poggiolesi@unifi.it 